\title{
Sublattice asymmetry of impurity doping in graphene: A review
}

\author{
James A. Lawlor ${ }^{* 1}$ and Mauro S. Ferreira ${ }^{1,2}$
}

\author{
Review \\ Address: \\ ${ }^{1}$ School of Physics, Trinity College Dublin, Dublin 2, Ireland and \\ ${ }^{2}$ CRANN, Trinity College Dublin, Dublin 2, Ireland \\ Email: \\ James A. Lawlor* - jalawlor@tcd.ie \\ * Corresponding author \\ Keywords: \\ band gap; electronic transport; graphene; nitrogen doping; sublattice \\ asymmetry
}

Beilstein J. Nanotechnol. 2014, 5, 1210-1217.

doi:10.3762/bjnano.5.133

Received: 29 April 2014

Accepted: 08 July 2014

Published: 05 August 2014

This article is part of the Thematic Series "Atomic scale interface design and characterisation: Theory - Electronic properties".

Guest Editor: A. Krasheninnikov

(C) 2014 Lawlor and Ferreira; licensee Beilstein-Institut.

License and terms: see end of document.

\begin{abstract}
In this review we highlight recent theoretical and experimental work on sublattice asymmetric doping of impurities in graphene, with a focus on substitutional nitrogen dopants. It is well known that one current limitation of graphene in regards to its use in electronics is that in its ordinary state it exhibits no band gap. By doping one of its two sublattices preferentially it is possible to not only open such a gap, which can furthermore be tuned through control of the dopant concentration, but in theory produce quasiballistic transport of electrons in the undoped sublattice, both important qualities for any graphene device to be used competetively in future technology. We outline current experimental techniques for synthesis of such graphene monolayers and detail theoretical efforts to explain the mechanisms responsible for the effect, before suggesting future research directions in this nascent field.
\end{abstract}

\section{Review \\ Introduction}

With its excellent transport properties and low dimensionality, graphene, an atomically thin layer of Carbon atoms bonded together in a hexagonal lattice, initially seems a strong candidate for use in many future commercial applications such as ultra high-speed transistors, integrated circuits and other novel devices [1-3]. One of the main problems with using regular graphene for such applications is the absence of a band gap in the electronic band structure [4], and as a result any field effect transistors (FETs) made using the material (so-called GFETs) would be unable to be switched off, rendering it useless as a logic device [5-7]. The most natural way to approach this issue is therefore to introduce a sizeable band gap and hence allowing more control over the current flow.

Although several methods exist to induce a band gap, for example 1D quantum confinement by construction of graphene 
nanoribbons (GNRs) [8], stacking of monolayers with perpendicular electric fields [6,7], strain [9] and mounting on a substrate [10-12], these methods are not without problems. Alternatives are therefore sought after with the minimum standard to meet or exceed the limits of silicon semiconductor technology, which is characterised by a current on/off ratio of roughly $10^{4}-10^{7}$ and a band gap of at least $340 \mathrm{meV}$ whilst maintaining high carrier mobility $[5,13,14]$.

Alteration of the crystal structure through the introduction of foreign dopants is one of the more realistic avenues of approach in realising this goal. Atomic dopants like boron (B) or nitrogen $(\mathrm{N})$ are a similar size to carbon and can be introduced easily in a variety of graphene growth processes, typically replacing carbon sites and forming substitutional impurities in the lattice, positively (p-) and negatively (n-) doping the system for $\mathrm{B}$ and $\mathrm{N}$ dopants respectively [15]. Early theoretical attempts at investigating the electronic properties of such a material found that a periodic arrangement of B or $\mathrm{N}$ dopants, forming a dopant superlattice, would open a band gap [16], but that a random distribution of dopants among lattice sites yields no band gap [17]. Further investigations using vacancies, where carbon atoms are removed from the lattice, found that both superlattices [18] and random distributions restricted to one of the two graphene sublattices [19] both lead to a tunable band gap, and in the latter case an emergence of magnetic properties in the system $[20,21]$.

Whilst these findings are certainly interesting, their scalability, and hence commercial application, is prohibited by the standard of precision that must be met in order to produce such materials. Circumventing these problems using nitrogen doped graphene has only come about relatively recently which we detail in the following section.

\section{A brief history of nitrogen doped graphene}

Exploiting the effects of nitrogen dopants on the transport properties of graphene has been an interesting experimental research topic for the last 5 years [22], whereby the methods and techniques to achieve this have been developed to include chemical vapour deposition (CVD), using $\mathrm{NH}_{3}$ as a precursor, arc discharge [23], embedded nitrogen and carbon sources within a metal substrate [24], ion implantation [25,26], ammonia [27] or nitrogen plasma $[28,29]$ treatments, and capable of achieving nitrogen dopant concentrations of up to around 10\% [30] and with direct applicablity to GFET technology and bio-sensing [28]. Although CVD is one of the more challenging methods, it seems the most reliable option and yields the best quality nitrogen doped graphene sheets [31] and single continuous sheets can be synthesised on the centimeter scale [32]. Using nitrogen dopants alone through CVD can yield bandgaps up to
$200 \mathrm{meV}$ [33] and by inclusion of boron co-dopants, through a tailored growth process, this can be expanded to around $600 \mathrm{meV}$ with $6 \%$ total dopant concentration [12]. Boron doping alone has shown to also open a band gap, tunable with dopant concentration $[34,35]$. This method results in small B-N domains embedded in the graphene sheet, so the precise mechanism behind the band gap opening may not be the same as that seen in a random B-N ensemble. Although these gaps are quite sizeable, the effect of scattering would lead to a detrimental impact on the transport characteristics. However, the recent theoretical prediction of sublattice asymmetry, a situation where dopants are distributed in one sublattice only, is expected to yield very low scattering transport properties. The electron wavefunctions in such a system are shown to mainly exist on the sublattice without dopants and can travel almost unhindered [36,37], which is very promising for overcoming the scattering problem.

It was not until 2011 that a viable experimental approach was found by Zhao et al. [38]. They discovered that graphene grown via chemical vapour deposition (CVD) in the presence of ammonia $\left(\mathrm{NH}_{3}\right)$ naturally incorporates nitrogen atoms as substitutional so-called 'graphitic' dopants (see Figure 1A) into the crystal, and with a distinct sublattice segregation of dopants. Indeed further research has uncovered a less pronounced asymmetry phenomenon using graphene implanted with nitrogen impurities followed by a high temperature annealing process [25], and it seems reasonable that there is a common mechanism with the CVD method. Due to the limited published work on this implantation and annealing method, our main focus will be on CVD and it is here that we begin our main discussions. We begin with a section covering in-depth the experimental work done to date, followed by a section on theoretical aspects and ending with a future outlook and conclusions.

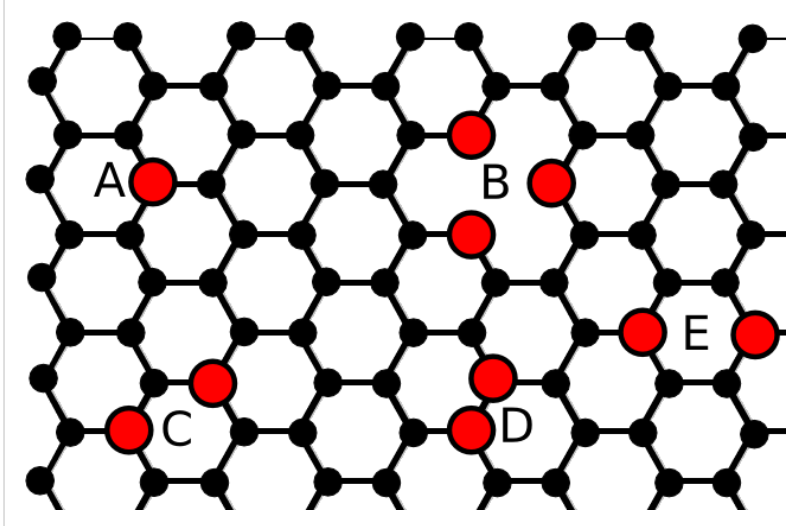

Figure 1: Schematic of a graphene lattice with the most common experimentally observed species of substitutional nitrogen dopants: (A) a single graphitic, (B) three pyridinics, (C) one $\mathrm{N}_{2}{ }^{A A}$ pair, (D) one $\mathrm{N}_{2}{ }^{A B}$ pair and (E) one $\mathrm{N}_{2}{ }^{A B^{\prime}}$ pair. 


\section{Experimental observation of sublattice asym- metry of $\mathrm{N}$-graphene}

The main focus of the work by Zhao et al. was the observation and characterization of nitrogen dopants via scanning tunnel spectroscopy (STS), followed by a short investigation on the transport properties of the resulting graphene. Their experimental observation of same sublattice segregation, at least on local scales, was noted as a curiosity but was not discussed in depth. A key finding by the researchers was that by varying the ammonia precursor concentration they could adjust the resulting embedded dopant concentration. Further theoretical work confirmed that a tunable band gap would be obtainable using this method [36], and along with the findings of Zhao et al. this sparked further interest into this phenomenon. It should be noted that the experimental conditions were a high vacuum CVD process on a $\mathrm{Cu}(111)$ substrate with a temperature of $1000{ }^{\circ} \mathrm{C}$, using $\mathrm{CH}_{4}$ and $\mathrm{NH}_{3}$ as graphene and nitrogen precursors respectively for $18 \mathrm{~min}$ reaction time achieving a dopant concentration of $0.3 \%$.

Shortly after this work a more focussed investigation was undertaken by Lv et al. [39]. They were able to replicate the findings of the first group and synthesised large areas of $\mathrm{N}$-doped monolayer graphene with the additional finding that by increasing the pressure in the growth phase from a high vacuum to atmospheric-pressure CVD would result in an abundance of so-called $\mathrm{N}_{2}{ }^{\mathrm{AA}}$ pairs [39] (see Figure 1C), which are thought to be a result of a higher number of intermolecule collisions during formation. Using the same substrate and precursors, they were also able to find that a minimum reaction time of $5 \mathrm{~min}$ and temperatures of $800{ }^{\circ} \mathrm{C}$ were optimum for the $\mathrm{N}$-doped graphene synthesis and moreover it was found that singular nitrogen dopants are more frequently found for reaction times below 10 min with the ratio of $\mathrm{N}_{2}{ }^{\mathrm{AA}}$ to single $\mathrm{N}$ increasing with reaction time. The samples had a typical concentration of around $0.25 \%$ nitrogen dopants.

More recently the addition of boron dopants into the lattice and their effects have been studied [40] using high vacuum CVD growth and adding $\mathrm{B}_{2} \mathrm{H}_{6}$ diborane instead of ammonia as the dopant precursor, achieving concentrations on the order of $0.3 \%$. The researchers compared B-doped and N-doped systems in detail and found no detectable sublattice asymmetry in the case of B-doped systems and thus the dopants were distributed evenly between sublattices. The reason for this is thought to be due to a strong interaction between the $\mathrm{Cu}(111)$ substrate and the boron impurity, in contrast to a very weak interaction with the substrate for nitrogen impurities [40].

The most recent and thorough study of asymmetric nitrogen dopants in graphene is by Zabet-Khosousi et al. [41] who again used the CVD growth process but with a pyridine $\left(\mathrm{C}_{5} \mathrm{H}_{5} \mathrm{~N}\right)$ precursor for the nitrogen and graphene instead of the conventional ammonia/methane mix. Figure 2 shows a rather striking STM image of a large area of N-doped graphene grown using this method, showing two clearly defined domains of different sublattice preference where the sharp borders are thought to arise from terracing of the substrate disrupting the asymmetry effect. The method was tailored to use a very smooth $\mathrm{Cu}(111)$ substrate and so they were able to achieve domain sizes far larger than previous efforts. Typical concentrations found with this method were around $0.2 \%$, very similar to the previous ammonia/methane method which points to an equivalent growth mechanism. By comparing N-doped graphene systems synthesised with this method to other systems made via nitrogen ion bombardement and ammonia post-treatment of a pristine graphene sheet, where no sublattice asymmetric configurations were found, it was suggested that the sublattice asymmetry must be occuring during formation of the graphene sheet. This is supported by the recent experiments using ion bombardement followed by high temperature annealing by Telychko et al. [25], where the impurities may be reconfiguring themselves locally during cooling. This phenomenon is not seen without the annealing process [26].

The common elements of all the aforementioned experimental reports are that they all use a $\mathrm{Cu}(111)$ substrate, all have reaction times exceeding $10 \mathrm{~min}$ in a temperature range of 800-900 ${ }^{\circ} \mathrm{C}$, and all have used either ammonia or pyridine precursors to achieve concentrations of up to $0.3 \%$. A review of other methods for $\mathrm{N}$-doped graphene synthesis finds no mention of sublattice asymmetry [29,30], it is known that this effect is mainly detectable through careful scanning tunnel microscopy of large areas. The most natural explanation for this lack of observation is then either that it is not being observed due to simply not looking, or that sublattice asymmetry is not a very robust effect and therefore requires a careful choice of synthesis procedure. Research into the mechanisms behind the phenomenon is therefore paramount in answering this question and could shed light on whether other methods or even dopant species are at all possible and this leads us naturally to a review of the current theoretical models of the effect.

\section{Theoretical models of the segregation effect}

It is clear that any degree of asymmetry between the two equivalent sublattices is the result of a symmetry breaking operation. Current theoretical attempts to explain the sublattice asymmetry in the nitrogen doping seen in the experiments discussed in the previous section suggest that the effect comes from either the energetically preferable positioning of nitrogen on the graphene edge during the growth process [41,42], where the symmetry breaking effect is the edge structure, or from 


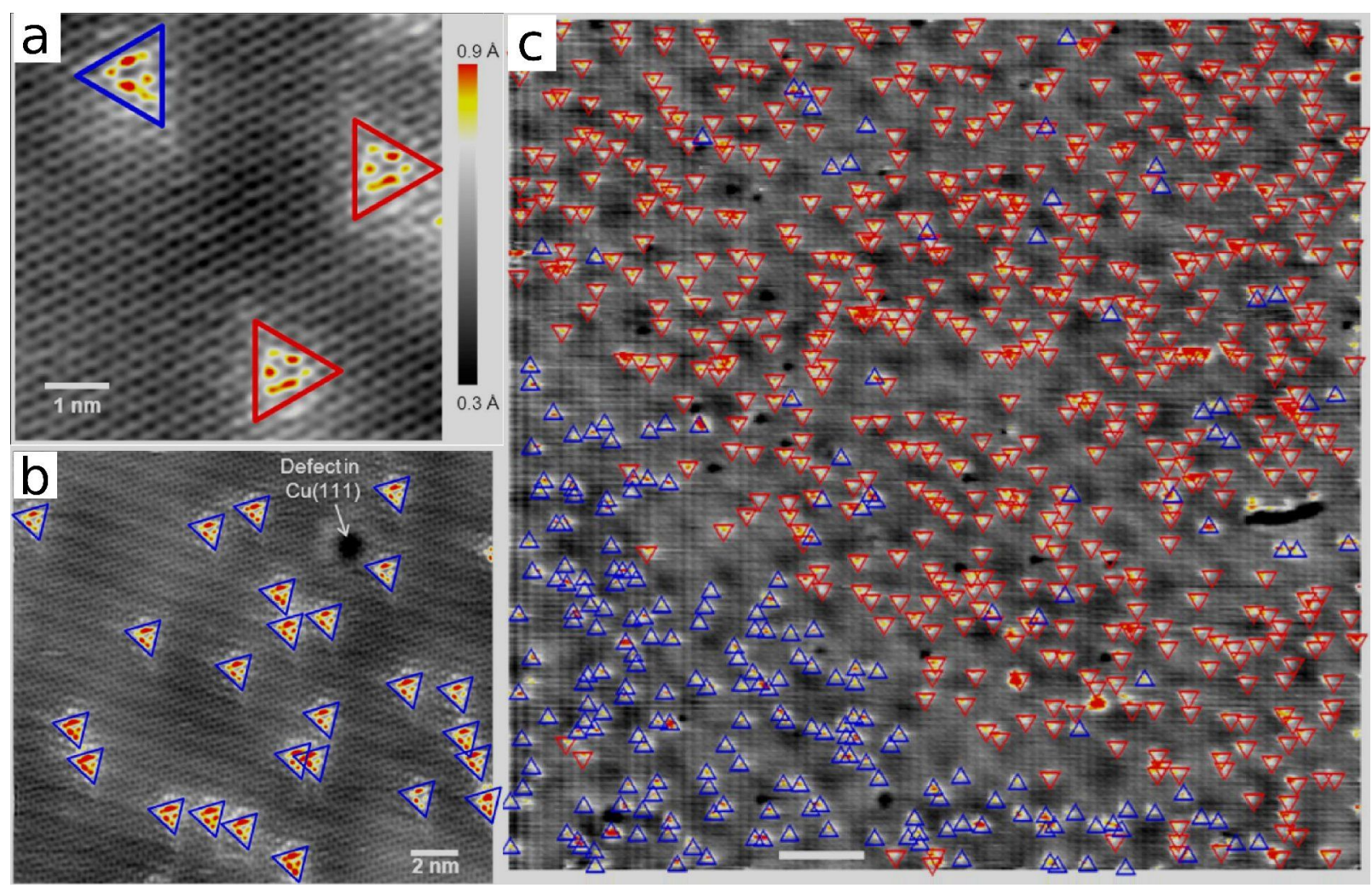

Figure 2: STM images of nitrogen doped graphene on (a) $7 \mathrm{~nm}^{2}$, (b) $20 \mathrm{~nm}^{2}$ and (c) $100 \mathrm{~nm}^{2}$ scales, adapted with permission from Zabet-Khosousi et al. [41]. Copyright 2014 American Chemical Society. The dopants locations are identified by finding bright spots on the STM image, corresponding to slight perturbations in the positions of the neighbouring carbon atoms. The dopant sublattice can be found through the orientation of the bright triangle features, where opposite sublattices appear as mirror images of each other as demonstrated in (a). The red and blue triangles in each subfigure correspond to impurities on the different sublattices. Part (c) best demonstrates that although the distribution of dopants appears random, when their sublattice is identified we see two very large domains appear with opposite sublattice segregation. Shown at the bottom, off-center left of (c) is a white $10 \mathrm{~nm}$ scalebar.

inter-impurity interactions in the impurity ensemble [39,43] where the symmetry is broken by the impurities themselves. These models will hereafter be referred to as the edge growth and interaction models respectively.

The edge growth model suggests that during the growth phase the most energetically favourable position for a nitrogen dopant being incorporated into a graphene edge would be that which leads to a same sublattice configuration for all impurities in a domain. Through density functional theory (DFT) calculations involving a graphene nanoribbon on a $\mathrm{Cu}(111)$ substrate, aiming to reproduce experimental conditions, a thorough investigation into the energetic favourable position of single graphitic nitrogens in the GNR was undertaken and the energetic difference between placing the nitrogen at one edge site over another on the opposite sublattice was found to be a substantial $1.3 \mathrm{eV}$. One drawback of this method is that one would expect therefore that the clearly defined segregation domains seen experimentally (see Figure 2 and [41]) would naturally fall in with the graphene grain boundaries, but this has not been observed. The role of inter-impurity interactions has been considered as an alternative, through both tight binding [43] and DFT [39,44] formalisms, although with differing conclusions. The tight binding method by Lawlor et al. [43] was part of a more in-depth theoretical investigation, and suggests that a Friedel oscillation-like perturbation in the long-range inter-impurity interactions [45] arise from adding impurities to the system, leading to a shift in the system's Fermi energy. Such a mechanism would explain both the non-commensurability between crystal grain boundaries and segregation domains, and would suggest why the alternative synthesis method of nitrogen implantation followed by high temperature annealing also results in impurities preferring to occupy the same sublattice [25]. Furthermore, the authors predict that as a result of this interaction and the reduction in nearest neighbour distance with increasing concentration, there exists a critical dopant concentration beyond which no sublattice asymmetry would be observed, as the energetic minimum occurs when the impurities are distributed evenly between sublattices. Although the exact value of this critical concentration is dependant on 
how the impurity is parameterised within the tight binding regime, the authors predict it to lie between $0.1 \%$ and $0.8 \%$. Comparing to the highest experimentally reported doping concentrations for samples with sublattice asymmetry $(0.3 \%)$, it is a hope that further experiments can clarify whether such a critical concentration exists. If the prediction of a critical concentration is accurate it would limit the band gap of a segregated device to around $100 \mathrm{meV}$ [36]. This figure, however, comes from matching tight binding and DFT band structure results, a method which is known to systematically underestimate such band gaps. Nevertheless, the band gap obtained can be expected to be much below that required for a GFET device.

In-depth DFT calculations by Hou et al. [44] found that the interactions between nitrogen impurities are generally repulsive in nature. More specifically, when the two impurities are placed close together the system energy is minimised for opposite sublattice configurations, with the exception of $\mathrm{N}_{2} \mathrm{AB}$. This contradicts the experimental reports of Lv et al. [39] who found an abundance of $\mathrm{N}_{2}{ }^{\mathrm{AA}}$ defects in their samples. A simplistic tight binding approach predicts that two identical impurities in close proximity to one another would indeed have an energetic minimum when both occupy the same sublattice [46], however this method ignores coulombic interaction which will be dominant at close separations. Interestingly, the DFT approach of Lv et al. of such nitrogen pairs finds that the lowest energy configuration would be $\mathrm{N}_{2}{ }^{\mathrm{AB}^{\prime}}$ (see Figure 1), approximately $0.3 \mathrm{eV}$ lower than that of $\mathrm{N}_{2}{ }^{\mathrm{AA}}$. This appears to be in disagreement with experiments where $\mathrm{N}_{2}{ }^{\mathrm{AA}}$ is more commonly found than $\mathrm{N}_{2}{ }^{A B^{\prime}}$. As a final note their calculations have also shown that two $\mathrm{N}_{2}{ }^{\mathrm{AA}}$ pairs have a lower energy when they share the same sublattice, however further studies have shown that the overall energy change resulting from an $\mathrm{N}_{2}{ }^{\mathrm{AA}}$ pair in the sheet is very high [42]. It is possible that these impurities also come from edge growth, but the controllable presence of $\mathrm{N}_{2} \mathrm{AA}$ in nitrogen ion implanted graphene [25] indicates again that this may not be the full picture as in this case the complete graphene sheet is already fabricated.

\section{Predicted electronic properties}

The earliest attempts to study nitrogen dopants and their effect on the graphene electronic properties were purely theoretical. Beginning with impurity superlattices, this research preceded the experimental realistation of nitrogen doped graphene. By introducing a controlled periodic arrangement of boron and nitrogen impurities a band gap was seen to open [16], and although such superlattice structures were not feasible on largescale it was further shown that a random distribution on one sublattice can also open a gap [37]. Further DFT studies showed that the band gap will increase with dopant concentration $[36,47]$ and that a dopant level of over $8 \%$, where impurities are all on the same sublattice, will produce a band gap of around $550 \mathrm{meV}$ far surpassing the minimum required for a CMOS $[14,36]$ and finding that the band gap scales with concentration to the power 3/4, as shown in Figure 3.

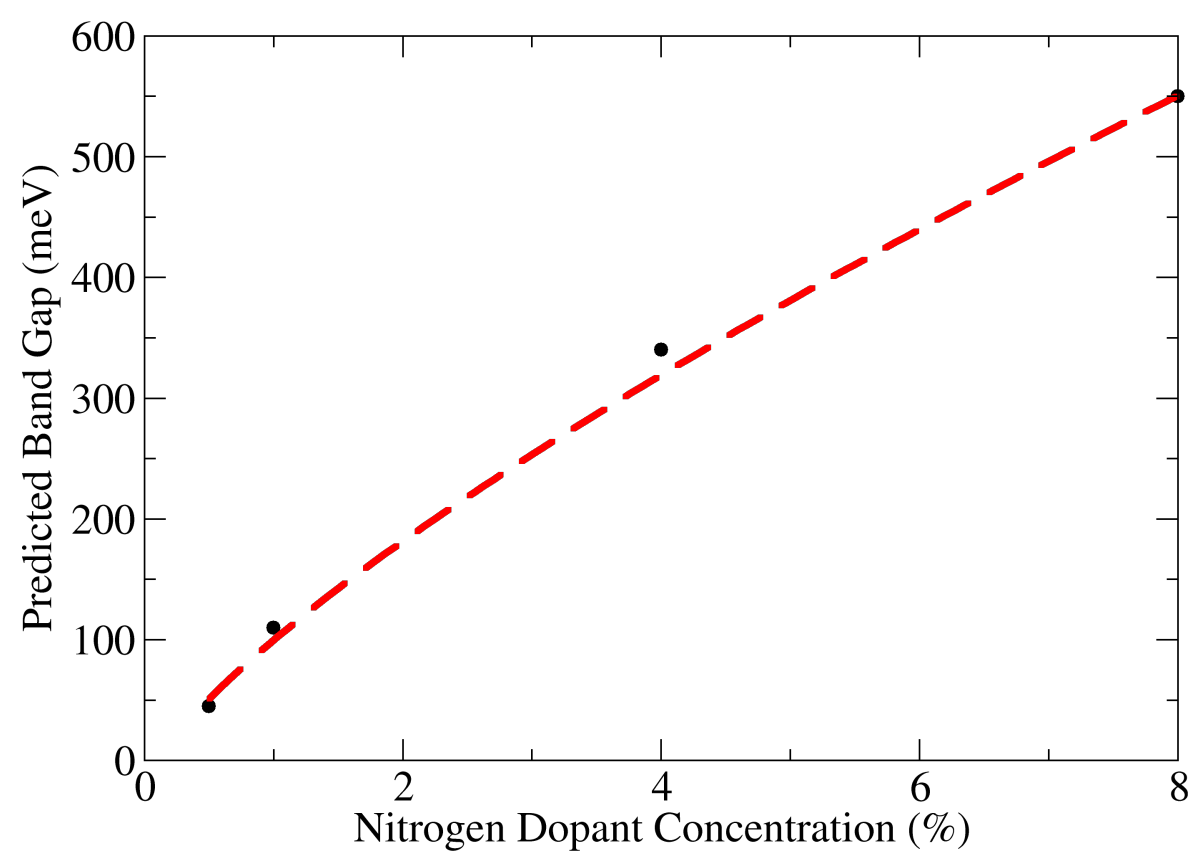

Figure 3: Predicted band gap against nitrogen dopant concentration. Black circles are calculated values from [36] and the red dashed line shows the expected band gap scaling with concentration, according to the power $3 / 4$ as discussed in the text. 
Even with a 4:1 doping ratio between sublattices, the band gap, although smaller, was shown to still exist. This is promising for scaleability where perfect asymmetric doping may not always be realizable. Beyond the realization of quasi-ballistic electron transport, sublattice segregated systems can also be used to induce magnetism $[19,48-50]$ and produce spin-polarized current $[51,52]$.

Much work has been done studying how the placement of dopants affects the properties of nanoribbons. DFT approaches, using a periodic system of dopants [53,54], and a more general Kubo-Greenwood approach [37] have shown that electron transport is enhanced when dopants are placed on one sublattice, compared to a random distribution, and that a band gap does indeed open. The difference in transport qualities between the asymmetrically doped versus completely randomly doped systems is illustrated by a conductance plot in Figure 4, where it is evident that the electrons in the former kind of device will be subjected to less scattering leading to an increase in the quantum conductance, closer to that of the pristine system when subjected to a positive bias.

It should also be noted that that symmetry breaking in nanoribbons occurs via edge effects and also results in a favourable sublattice [58], however the mechanism is distinctly different from that in graphene.

\section{Outlook}

At the time of writing, the synthesis of sublattice asymmetric graphene is only just now becoming practical. There are still many unanswered questions and the most important ones we outline in this section.

The natural question to ask is if this asymmetry is particular to nitrogen only, or if it can be found with other dopants. Theoretical findings in the past have shown that such an effect can be expected with a dilute concentration of certain adsorbates [59], and indeed more recent studies suggest that this could also be possible with other impurities [43]. Whilst it is known experimentally that molybdenum impurities exhibit same sublattice configurations in bilayer epitaxial graphene, the mechanism behind this is not currently understood [60]. Boron has been studied in a similar CVD growing regime to nitrogen where it has been proposed that the role of the substrate can play a critical role in the manifestation of segregation. In this case, it is thought that the strong interactions between the $\mathrm{Cu}(111)$ crystal and the boron dopants destroy any asymmetry effects [40]. It is then logical to ask whether suitable substrates can be identified to produce segregated boron doped graphene sheets, which would have the effect of p-doping the system, and then to ask whether this can be extended to other species of dopants. We note that it is known that there are weak interactions between graphene and $\mathrm{Al}, \mathrm{Ag}, \mathrm{Au}, \mathrm{Pt}(111)$ substrates, all of which leave

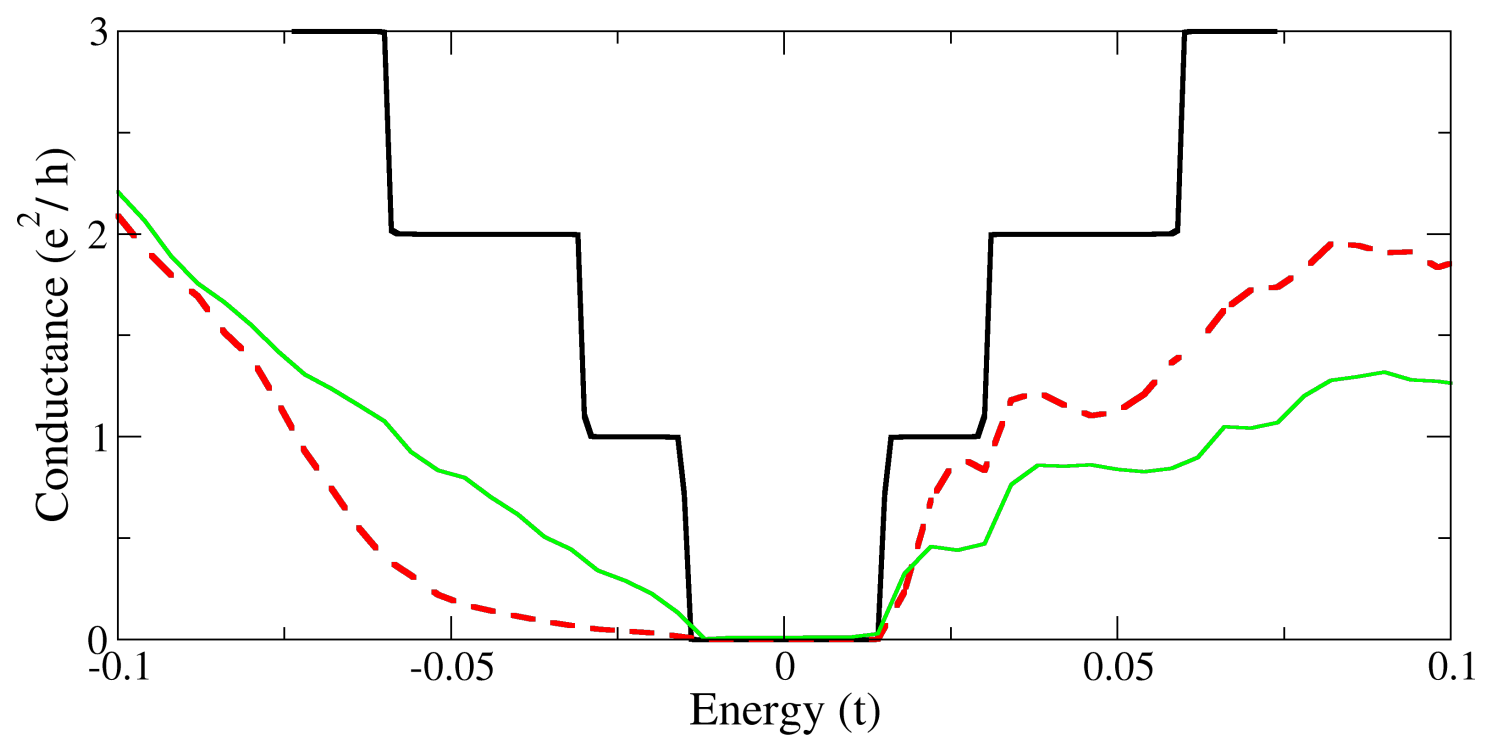

Figure 4: Quantum conductance through a $15 \mathrm{~nm}$ wide graphene nanoribbon with a $7.5 \mathrm{~nm}$ long scattering region containing a dispersion of substitutional nitrogen impurities, in a similar vein to the method of Botello-Mendez et al. [37], calculated using a recursive Green's Function method [55,56], the Kubo formula for conductance [57] and a configurational average of 50 systems. Energy is in units of the tight binding nearest neighbour hopping energy between carbon atoms, $t=2.7 \mathrm{eV}$. Shown is the predicted conductance for pristine (black), randomly doped (green solid) and single sublattice doped (red dashed) systems, where a dopant concentration of $1 \%$ nitrogen was used. 
the electronic structure intact [61], whilst substrates such as $\mathrm{Ni}$ have considerably stronger interactions [62]. Another way to investigate the presence of sublattice asymmetry with dopants other than nitrogen is via ion bombardement [63], which could be combined with the high temperature annealing process discussed previously.

The experimental realisation of spin-polarized transport should also be pursued, and could spark additional interest in this research area beyond quasi-ballistic transport. Investigation of the effects of strain on such a system is difficult due to the technicalities of the CVD method, but could be explored using adsorbates instead of substitutions or by incorporation of the strain in to the nitrogen ion bombardement and annealing procedure discussed earlier. Another open question is if a critical concentration of dopants exists, as mentioned in the theory section, which would limit the available bandgap below the threshold needed for future use in GFET devices. Currently all experimental reports on sublattice asymmetry have very low concentrations of dopants around $0.3 \%$, so it should be feasible to test the existence of a critical concentration of using available methods [30]. Consequentially this would shed more light on the mechanism responsible for the segregation, and whether in fact the inter-impurity interactions and edge growth effects are complementary effects.

\section{Conclusion}

This paper reviews the current state of experimental and theoretical research in sublattice asymmetric nitrogen doped graphene. Not only are such systems now able to be synthesised in the lab, we have seen that this area shows promise attaining graphene-based FET devices due to the opening of a bandgap whilst maintaining the excellent transport properties of graphene, something which is not realisable in graphene with no sublattice imbalance in dopant distribution. While there are still many open questions in the field these should be answerable within the scope of current techniques.

\section{Acknowledgements}

The authors acknowledge financial support from the Programme for Research in Third Level Institutions (PRTLI), Science Foundation Ireland (Grant No. SFI 11/ RFP.1/MTR/ 3083).

\section{References}

1. Geim, A. K.; Novoselov, K. S. Nat. Mater. 2007, 6, 183-191. doi:10.1038/nmat1849

2. Novoselov, K. S.; Fal'ko, V. I.; Colombo, L.; Gellert, P. R.; Schwab, M. G.; Kim, K. Nature 2012, 490, 192-200. doi:10.1038/nature11458

3. Chen, J.-H.; Jang, C.; Xiao, S.; Ishigami, M.; Fuhrer, M. S. Nat. Nanotechnol. 2008, 3, 206-209. doi:10.1038/nnano.2008.58
4. Allen, M. J.; Tung, V. C.; Kaner, R. B. Chem. Rev. 2010, 110, 132-145. doi:10.1021/cr900070d

5. Schwierz, F. Nat. Nanotechnol. 2010, 5, 487-496. doi:10.1038/nnano.2010.89

6. Lin, Y.-M.; Jenkins, K. A.; Valdes-Garcia, A.; Small, J. P.; Farmer, D. B.; Avouris, P. Nano Lett. 2009, 9, 422-426. doi:10.1021/nl803316h

7. Zhang, Y.; Tang, T.-T.; Girit, C.; Hao, Z.; Martin, M. C.; Zettl, A.; Crommie, M. F.; Shen, Y. R.; Wang, F. Nature 2009, 459, 820-823. doi:10.1038/nature08105

8. Li, X.; Wang, X.; Zhang, L.; Lee, S.; Dai, H. Science 2008, 319, 1229-1232. doi:10.1126/science.1150878

9. Naumov, I. I.; Bratkovsky, A. M. Phys. Rev. B 2011, 84, 245444. doi:10.1103/PhysRevB.84.245444

10. Novoselov, K. Nat. Mater. 2007, 6, 720-721. doi:10.1038/nmat2006

11. Zhou, S. Y.; Gweon, G.-H.; Fedorov, A. V.; First, P. N.; de Heer, W. A.; Lee, D.-H.; Guinea, F.; Castro Neto, A. H.; Lanzara, A. Nat. Mater. 2007, 6, 770-775. doi:10.1038/nmat2003

12. Chang, C.-K.; Kataria, S.; Kuo, C.-C.; Ganguly, A.; Wang, B.-Y.; Hwang, J.-Y.; Huang, K.-J.; Yang, W.-H.; Wang, S.-B.; Chuang, C.-H.; Chen, M.; Huang, C.-I.; Pong, W.-F.; Song, K.-J.; Chang, S.-J.; Guo, J.-H.; Tai, Y.; Tsujimoto, M.; Isoda, S.; Chen, C.-W.; Chen, L.-C.; Chen, K.-H. ACS Nano 2013, 7, 1333-1341. doi:10.1021/nn3049158

13. Avouris, P. Nano Lett. 2010, 10, 4285-4294. doi:10.1021/nl102824h

14. Kim, K.; Choi, J.-Y.; Kim, T.; Cho, S.-H.; Chung, H.-J. Nature 2011, 479, 338-344. doi:10.1038/nature10680

15. Islam, M. S.; Ushida, K.; Tanaka, S.; Makino, T.; Hashimoto, A. Comput. Mater. Sci. 2014, in press. doi:10.1016/j.commatsci.2014.01.040

16. Casolo, S.; Martinazzo, R.; Tantardini, G. F. J. Phys. Chem. C 2011, 115, 3250-3256. doi:10.1021/jp109741s

17. Lherbier, A.; Blase, X.; Niquet, Y.-M.; Triozon, F.; Roche, S. Phys. Rev. Lett. 2008, 101, 036808. doi:10.1103/PhysRevLett.101.036808

18. Martinazzo, R.; Casolo, S.; Tantardini, G. F. Phys. Rev. B 2010, 81, 245420. doi:10.1103/PhysRevB.81.245420

19. Palacios, J. J.; Fernández-Rossier, J.; Brey, L. Phys. Rev. B 2008, 77, 195428. doi:10.1103/PhysRevB.77.195428

20. Cresti, A.; Ortmann, F.; Louvet, T.; Van Tuan, D.; Roche, S. Phys. Rev. Lett. 2013, 110, 196601. doi:10.1103/PhysRevLett.110.196601

21. Pereira, V. M.; Lopes dos Santos, J. M. B.; Castro Neto, A. H. Phys. Rev. B 2008, 77, 115109. doi:10.1103/PhysRevB.77.115109

22. Wei, D.; Liu, Y.; Wang, Y.; Zhang, H.; Huang, L.; Yu, G. Nano Lett. 2009, 9, 1752-1758. doi:10.1021/nl803279t

23. Guan, L.; Cui, L.; Lin, K.; Wang, Y. Y.; Wang, X. T.; Jin, F. M.; He, F.; Chen, X. P.; Cui, S. Appl. Phys. A 2011, 102, 289-294. doi:10.1007/s00339-010-6110-5

24. Zhang, C.; Fu, L.; Liu, N.; Liu, M.; Wang, Y.; Liu, Z. Adv. Mater. 2011, 23, 1020-1024. doi:10.1002/adma.201004110

25. Telychko, M.; Mutombo, P.; Ondráček, M.; Hapala, P.; Bocquet, F. C.; Kolorenč, J.; Vondráček, M.; Jelínek, P.; Švec, M. ACS Nano 2014, 8, 7318-7324. doi:10.1021/nn502438k

26. Bangert, U.; Pierce, W.; Kepaptsoglou, D. M.; Ramasse, Q.; Zan, R.; Gass, M. H.; Van den Berg, J. A.; Boothroyd, C. B.; Amani, J.; Hofsäss, H. Nano Lett. 2013, 13, 4902-4907. doi:10.1021/nl402812y

27. Lin, Y.-C.; Lin, C.-Y.; Chiu, P.-W. Appl. Phys. Lett. 2010, 96, 133110. doi:10.1063/1.3368697

28. Wang, Y.; Shao, Y.; Matson, D. W.; Li, J.; Lin, Y. ACS Nano 2010, 4, 1790-1798. doi:10.1021/nn100315s 
29. Joucken, F.; Tison, Y.; Lagoute, J.; Dumont, J.; Cabosart, D.; Zheng, B.; Repain, V.; Chacon, C.; Girard, Y.; Botello-Mendez, A. R.; Rousset, S.; Sporken, R.; Charlier, J.-C.; Henrard, L. Phys. Rev. B 2012, 85, 161408. doi:10.1103/PhysRevB.85.161408

30. Wang, H.; Maiyalagan, T.; Wang, X. ACS Catal. 2012, 2, 781-794. doi:10.1021/cs200652y

31. Wang, H.; Xie, M.; Thia, L.; Fisher, A.; Wang, X. J. Phys. Chem. Lett. 2014, 5, 119-125. doi:10.1021/jz402416a

32. Gao, H.; Song, L.; Guo, W.; Huang, L.; Yang, D.; Wang, F.; Zuo, Y.; Fan, X.; Liu, Z.; Gao, W.; Vajtai, R.; Hackenberg, K.; Ajayan, P. M. Carbon 2012, 50, 4476-4482. doi:10.1016/j.carbon.2012.05.026

33. Usachov, D.; Vilkov, O.; Grüneis, A.; Haberer, D.; Fedorov, A.; Adamchuk, V. K.; Preobrajenski, A. B.; Dudin, P.; Barinov, A.; Oehzelt, M.; Laubschat, C.; Vyalikh, D. V. Nano Lett. 2011, 11, 5401-5407. doi:10.1021/nl2031037

34. Tang, Y.-B.; Yin, L.-C.; Yang, Y.; Bo, X.-H.; Cao, Y.-L.; Wang, H.-E.; Zhang, W.-J.; Bello, I.; Lee, S.-T.; Cheng, H.-M.; Lee, C.-S. ACS Nano 2012, 6, 1970-1978. doi:10.1021/nn3005262

35. Fan, X.; Shen, Z.; Liu, A.; Kuo, J.-L. Nanoscale 2012, 4, 2157-2165. doi:10.1039/c2nr11728b

36. Lherbier, A.; Botello-Méndez, A. R.; Charlier, J.-C. Nano Lett. 2013, 13, 1446-1450. doi:10.1021/nl304351z

37. Botello-Méndez, A. R.; Lherbier, A.; Charlier, J. C. Solid State Commun. 2013, 175-176, 90-100. doi:10.1016/j.ssc.2013.08.029

38. Zhao, L.; He, R.; Rim, K. T.; Schiros, T.; Kim, K. S.; Zhou, H.; Gutiérrez, C.; Chockalingam, S. P.; Arguello, C. J.; Pálová, L.; Nordlund, D.; Hybertsen, M. S.; Reichman, D. R.; Heinz, T. F.; Kim, P.; Pinczuk, A.; Flynn, G. W.; Pasupathy, A. N. Science 2011, 333, 999-1003. doi:10.1126/science.1208759

39. Lv, R.; Li, Q.; Botello-Méndez, A. R.; Hayashi, T.; Wang, B.; Berkdemir, A.; Hao, Q.; Elias, A. L.; Cruz-Silva, R.; Gutiérrez, H. R.; Kim, Y. A.; Muramatsu, H.; Zhu, J.; Endo, M.; Terrones, H.; Charlier, J.-C.; Pan, M.; Terrones, M. Sci. Rep. 2012, 2, 586 doi:10.1038/srep00586

40. Zhao, L.; Levendorf, M.; Goncher, S.; Schiros, T.; Pálová, L.; Zabet-Khosousi, A.; Rim, K. T.; Gutiérrez, C.; Nordlund, D.; Jaye, C.; Hybertsen, M.; Reichman, D.; Flynn, G. W.; Park, J.; Pasupathy, A. N. Nano Lett. 2013, 13, 4659-4665. doi:10.1021/nl401781d

41. Zabet-Khosousi, A.; Zhao, L.; Pálová, L.; Hybertsen, M. S.; Reichman, D. R.; Pasupathy, A. N.; Flynn, G. W. J. Am. Chem. Soc. 2014, 136, 1391-1397. doi:10.1021/ja408463g

42. Deretzis, I.; La Magna, A. Phys. Rev. B 2014, 89, 115408. doi:10.1103/PhysRevB.89.115408

43. Lawlor, J. A.; Gorman, P. D.; Power, S. R.; Bezerra, C. G.; Ferreira, M. S. Carbon 2014, in press. doi:10.1016/j.carbon.2014.05.069

44. Hou, Z.; Wang, X.; Ikeda, T.; Terakura, K.; Oshima, M.; Kakimoto, M.-a.; Miyata, S. Phys. Rev. B 2012, 85, 165439. doi:10.1103/PhysRevB.85.165439

45. Lambin, P.; Amara, H.; Ducastelle, F.; Henrard, L. Phys. Rev. B 2012, 86, 045448. doi:10.1103/PhysRevB.86.045448

46. Lawlor, J. A.; Power, S. R.; Ferreira, M. S. Phys. Rev. B 2013, 88 , 205416. doi:10.1103/PhysRevB.88.205416

47. Rani, P.; Jindal, V. K. RSC Adv. 2012, 3, 802-812. doi:10.1039/C2RA22664B

48. Nair, R. R.; Sepioni, M.; Tsai, I.-L.; Lehtinen, O.; Keinonen, J.; Krasheninnikov, A. V.; Thomson, T.; Geim, A. K.; Grigorieva, I. V. Nat. Phys. 2012, 8, 199-202. doi:10.1038/nphys2183
49. Singh, R.; Kroll, P. J. Phys.: Condens. Matter 2009, 21, 196002. doi:10.1088/0953-8984/21/19/196002

50. Santos, E. J.; Sánchez-Portal, D.; Ayuela, A. Phys. Rev. B 2010, 81, 125433. doi:10.1103/PhysRevB.81.125433

51. Park, H.; Wadehra, A.; Wilkins, J. W.; Castro Neto, A. H. Phys. Rev. B 2013, 87, 085441. doi:10.1103/PhysRevB.87.085441

52. Rakyta, P.; Kormányos, A.; Cserti, J. Phys. Rev. B 2011, 83, 155439. doi:10.1103/PhysRevB.83.155439

53. Owens, J. R.; Cruz-Silva, E.; Meunier, V. Nanotechnology 2013, 24 , 235701. doi:10.1088/0957-4484/24/23/235701

54. Chen, T.; Li, X.-F.; Wang, L.-L.; Li, Q.; Luo, K.-W.; Zhang, X.-H.; Xu, L. J. Appl. Phys. 2014, 115, 053707. doi:10.1063/1.4863638

55. Economou, E. N. Green's functions in quantum physics; Springer, 1984; Vol. 3.

56. Lopez Sancho, M. P.; Lopez Sancho, J. M.; Rubio, J. J. Phys. F: Met. Phys. 1984, 14, 1205.

doi:10.1088/0305-4608/14/5/016

57. Kubo, R. Can. J. Phys. 1956, 34, 1274-1277. doi:10.1139/p56-140

58. Power, S. R.; de Menezes, V. M.; Fagan, S. B.; Ferreira, M. S. Phys. Rev. B 2009, 80, 235424. doi:10.1103/PhysRevB.80.235424

59. Cheianov, V. V.; Syljuåsen, O.; Altshuler, B. L.; Fal'ko, V. I. EPL 2010, 89, 56003. doi:10.1209/0295-5075/89/56003

60. Wan, W.; Li, H.; Huang, H.; Wong, S. L.; Lv, L.; Gao, Y.; Wee, A. T. S. ACS Nano 2013, 8, 970-976. doi:10.1021/nn4057929

61. Khomyakov, P. A.; Giovannetti, G.; Rusu, P. C.; Brocks, G.; van den Brink, J.; Kelly, P. J. Phys. Rev. B 2009, 79, 195425. doi:10.1103/PhysRevB.79.195425

62. Xu, Z.; Buehler, M. J. J. Phys.: Condens. Matter 2010, 22, 485301. doi:10.1088/0953-8984/22/48/485301

63. Wang, H.; Wang, Q.; Cheng, Y.; Li, K.; Yao, Y.; Zhang, Q.; Dong, C.; Wang, P.; Schwingenschlögl, U.; Yang, W.; Zhang, X. X. Nano Lett. 2011, 12, 141-144. doi:10.1021/nl2031629

\section{License and Terms}

This is an Open Access article under the terms of the Creative Commons Attribution License (http://creativecommons.org/licenses/by/2.0), which permits unrestricted use, distribution, and reproduction in any medium, provided the original work is properly cited.

The license is subject to the Beilstein Journal of Nanotechnology terms and conditions: (http://www.beilstein-journals.org/bjnano)

The definitive version of this article is the electronic one which can be found at: doi:10.3762/bjnano.5.133 